\title{
Comparative Evaluation of Microscopy \& Antigen Detection for Cryptosporidium by Elisa in Patients with Immunocompromised Status.
}

\author{
Anil kumar ${ }^{1}$, Roma Goyal ${ }^{2}$, Anil Kumar ${ }^{3}$, Paramjit Singh ${ }^{4}$ \\ ${ }^{\prime}$ (Department of Microbiology, L.L.R.M Medical College, Meerut, India) \\ ${ }^{2}$ (Department of Microbiology, Muzaffarnagar Medical College, Muzaffarnagar, India) \\ ${ }^{3}$ (Department of Microbiology, M.L.B. Medical College, Jhansi, India) \\ ${ }^{4}$ (Department of Microbiology, Muzaffarnagar Medical College, Muzaffarnagar, India)
}

\begin{abstract}
:
Background: Cryptosporidium is a protozoan parasite with worldwide distribution mainly affecting children and HIV infected individuals with low CD4 counts. The infection is self limiting in immunocompetent hosts but can be severe and persist in the immunocompromised and malnourished individuals. The aim of this study was to estimate the prevalence of Cryptosporidium infection in faeces among immunocompromised patients

Methods

Faecal samples from 113 immunocompromised patients with diarrhoea and without diarrhoea were examined for oocysts by microscopy and Cryptosporidium antigen test was performed using ELISA method.

Results

Of the 113 stool samples our study indicated, 20.3\% Cryptosporidium infection prevalence in the immunocompromised participated population. Furthermore, Modified ZN staining(Kinyon's) detected 13(11.5\%), wet mount by direct microscopy 2(1.7\%)and concentration method 4(3.53\%) stool samples positive for oocysts of Cryptosporidium parvum and Cryptococcal antigen by ELISA 23(20.3\%) Stool samples were positive.

Conclusion

The present study confirmed the high prevalence of Cryptosporidiumantigen in fecal samples of immunocompromised patients in the region. As no chemotherapeutic agents have yet proven, especially in immunosuppressed patients, therefore our results highlight the importance of preventive intervention in these groups.
\end{abstract}

Keywords: Cryptosporidium, Oocysts, Kinyon's staining Coproantigen, Immunocompromised patients, ELISA

\section{Introduction:}

Cryptosporidiosis is caused by Cryptosporidium strains, a parasite classified as emerging pathogens. The organism infects the gastrointestinal epithelium to produce a diarrhea that is self-limited in immunocompetent persons, but potentially life threating in immunocompromised persons (1). Furthermore, Cryptosporidium stated an organism with low infective dose and resistant to conventional water disinfectants (2). In addition, no definitive treatment and proven protocol has been achieved for cryptosporidiosis (3). Therefore, prevention seems to be the main effective disease protection, especially among immuno-compromised individuals.

Parasitic infections with Isospora belli, Entamoeba histolytica and Cryptosporidium parvum have been reported as being the most frequently identified organisms in India[4]. Cryptosporidium parvum is a small parasite measuring about $3-5 \mu \mathrm{m}$ in diameter. The life cycle of Cryptosporidium parvum is completed within the small intestine and colon of the host with the developing stages associated with the luminal surface of the intestinal epithelial cells where it remains intracellular but extracytoplasmic[3]. Transmission occurs by ingestion of contaminated food or water or contact with infected animals (beef, cattle, sheep, pig and dog). (5)

Cryptosporidium species are tiny microscopic parasites that cause cryptosporidiosis. Cryptosporidium parvum ingested as an oocyst, undergoes excystation and sporozoites parasitizes the host. The oocysts are highly resistant to common household disinfectants and survive for long periods in the environment [3]. Cryptosporidiosis is a major cause of diarrhoea in children with or without HIV infection in developing countries. AIDS and protein energy malnutrition are severely impairing the immune system, in such patients cellular immunity is low and implies that cellular immunity is the major component in eliminating the infection [6]. This is evident by correlation between lower CD4 T cells count, risk and severity of cryptosporidiosis [7]. It has major public health implication because infection can result from exposure to low doses of oocysts [7]. Identification of oocysts in stool samples have been described, these include modified Ziehl-Neelsen (ZN) 
staining (2\% sulphuric acid must be used) [8,9] Giemsa (GS) staining [10] and Sheather sugar flotation (SSF) technique [10-12]. The purpose of this study was to find out frequency of cryptosporidiosis, creating awareness among the population and to highlight the importance of detection of oocysts in routine stool examination and detection of Cryptococcal antigen by ELISA.

\section{Material and Methods}

A retrospective study was carried out in the Department of Microbiology for a period of one year, Overall, 113 immunocompromised patients including 9 with haematological malignancy, 32 renal transplant patients and 64 confirmed $\mathrm{HIV}^{+}$patients were also participated. The patients were interviewed and a questionnaire was completed for demographical, hygienic, and environmental life style with special emphasis on the gastrointestinal symptoms as diarrohea with duration, acute $(<2$ weeks) and chronic $(>4$ weeks $)$ and persistent (2-4 weeks). Duration of immunosuppressive and antiretroviral therapy, relevant other laboratory investigations and specific laboratory test, such as HIV- ELISA by 3 kits, CD-4 counts were studied.

The patients were provided a wide mouthed, clean, dry, properly labelled plastic container for collection of stool samples. Five grams of formed or about $10 \mathrm{ml}$ of unformed stool was collected and samples were examined within one to two hours of collection. Wet mount by both direct and concentration method was done from stool samples and smears were prepared stained by modified Ziehl-Neelsen staining method. [8,9] The stained smears were examined under oil for oocysts of Cryptosporidium parvum.

A total 113 stool samples were collected and stored at $-20^{\circ} \mathrm{C}$ without any preservative until used. The ELISA was used forCryptosporidium antigen detection in stool samples. The samples were defrosted and enzyme immunoassay was performed as described by the manufacturer. The results were carried out spectrophotometrically through ELISA reader according to manufacturer guidelines.

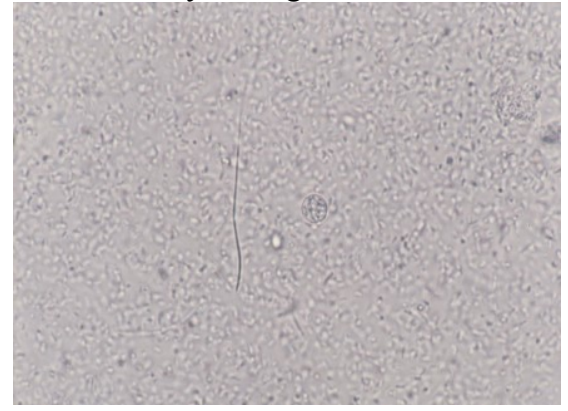

Figure 1: shows Cryptosporidium Oocyst Normal Saline mount

(40X)

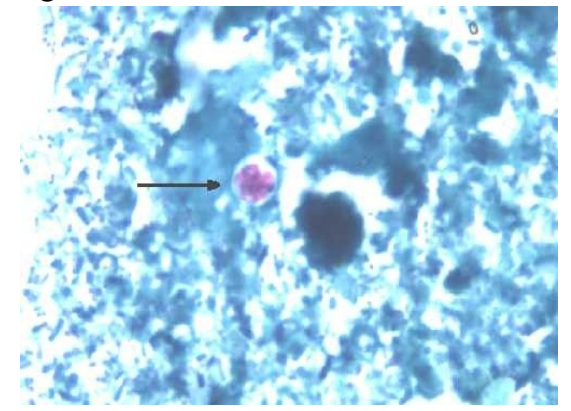

Figure 2: shows Cryptosporidium Oocyst, Modified Kinyon's Stain (100 X)

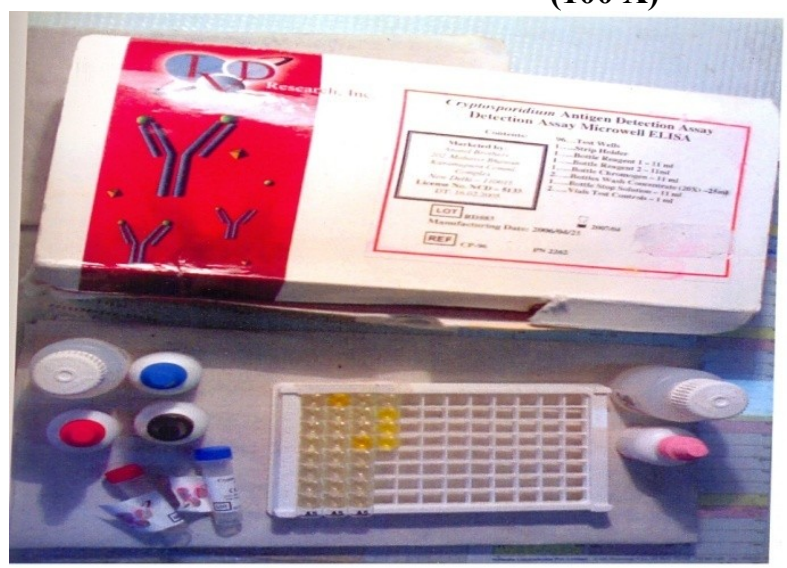

\section{figure 3: Crytosporidium ELISA Kit}

\section{RESULT}

Among 113 immunocompromised patients, were included in the study mean age of the patients was 39.5 years though the range varied from $3-76$ years. $77 \%$ of the patients were between $21-50$ years of age. A predominance of males( $76 \%)$. Over females $(24 \%)$ was observed male female ratio was 3:1. 
Among them $56.6 \%$ were from immunology ward followed by Nephrology(28\%), Haematology $(8 \%)$ and others (7\%). [Table- 1$]$

Oocysts of Cryptosporidium were detected on direct wet mount 2, in 4 after Concentration Method and on applying Kinyon's Staining 13 additional oocysts of Crytosporidium were detected[Table-2].All Oocyst of Cryptosporidium were detected from patients with diarrohea. Mixed infection with Oocyst of Crytosporidium was found in 2 patients each with Larvae of Strongyloides stercolaris and Isospora respectively.

Cryptosporidium antigen detection in stool was detected by ELISA in 23/113(21\%) as compared to microscopy in 13/113(11.5\%)[Table 3]. On subgroup analysis, it was found that out of 64 HIV positive patients, $7 / 8$ positive by microscopy were also positive by ELISA. $9 / 56$ patients negative by microscopy was positive by ELISA. All patients with Cryptosporidium has CD4 count less than 200 cell/ $\mu 1$ [Table 4]. Out of 32 Renal transplant recipient $4 / 5$ positive by microscopy were also positive by ELISA and 3/27 patients negative by microscopy were positive by ELISA[Table 5].Among 9 patients with Hematological malignancies and 8 others no opportunistic were detected from any case.

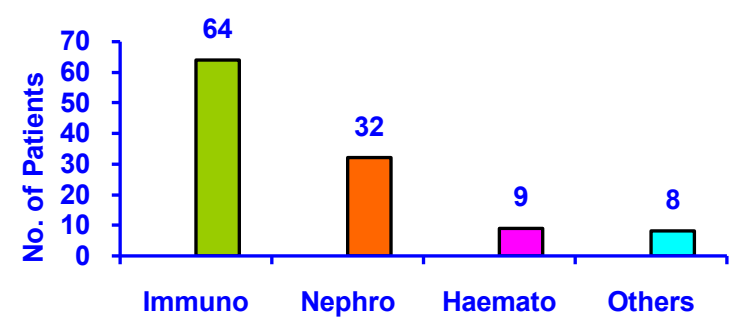

Table 1: Ward wise distribution of Immuno-compromised patients

\begin{tabular}{|c|c|c|c|c|c|}
\hline \multirow{2}{*}{ Microscopy } & \multicolumn{2}{|c|}{$(\mathbf{N}=113)$ Direct } & \multicolumn{2}{c|}{$(\mathbf{N}=113)$ Concentration method } \\
\cline { 2 - 6 } & Normal Saline & Iodine & Normal saline & Iodine & Kinyoun's \\
& 2 & 2 & 4 & 4 & 13 \\
& Cryptosporidium & Cryptosporidium & Cryptosporidium & Cryptosporidium & Cryptosporidium \\
\hline Negative & 111 & 111 & 109 & 109 & 100 \\
\hline
\end{tabular}

Table-2 : Comparison of direct and concentration method.

\begin{tabular}{|c|c|c|c|}
\hline \multirow{2}{*}{ Antigen } & \multicolumn{2}{|c|}{ Microscopy } & \multirow{2}{*}{ Total } \\
\cline { 2 - 3 } & Positive & Negative & 23 \\
\hline Positive & $11(\mathrm{a})$ & $12(\mathrm{~b})$ & 90 \\
\hline Negative & $2(\mathrm{c})$ & $88(\mathrm{~d})$ & $\mathbf{1 1 3}$ \\
\hline Total & $\mathbf{1 3}$ & $\mathbf{1 0 0}$ & \\
\hline
\end{tabular}

Table -3: Comparison of Microscopy and Cryptosporidium antigen detection in stool.

\begin{tabular}{|c|c|c|c|}
\hline \multirow{2}{*}{ Antigen } & \multicolumn{2}{|c|}{ Microscopy } & \multirow{2}{*}{ Total } \\
\cline { 2 - 4 } & Positive & Negative & 16 \\
\hline Positive & 7 & 9 & 48 \\
\hline Negative & 1 & 47 & $\mathbf{6 4}$ \\
\hline Total & $\mathbf{8}$ & $\mathbf{5 6}$ & \\
\hline
\end{tabular}

Table 4 : Comparative evaluation of microscopy with antigen detection in HIV

\begin{tabular}{|c|c|c|c|}
\hline \multirow{2}{*}{ Antigen } & \multicolumn{2}{|c|}{ Microscopy } & \multirow{2}{*}{ Total } \\
\cline { 2 - 4 } & Positive & Negative & 7 \\
\hline Positive & 4 & 3 & 25 \\
\hline Negative & 1 & 24 & $\mathbf{3 2}$ \\
\hline Total & $\mathbf{5}$ & $\mathbf{2 7}$ & 7 \\
\hline
\end{tabular}

Table 5 : Comparative evaluation of microscopy and antigen detection for Cryptosporidium in renal transplant recipients 


\section{DISCUSSION}

Cryptosporidiosis is recognized as an important gastroenteritis disorder of immunocompromised patients. In such cases, it may lead to high morbidity and mortality.[12] The present work was designed to study the spectrum of Cryptosporidium in immunocompromised patients at our centre. A total of 113 patients were included in the study. Of these 86 were males and 27 were females. The male female ratio was 3:1. Similar finding of male preponderance has been quoted by many workers.[14] They found a male and female ratio of $3: 1$ when detected Cryptosporidium infection in 22 patients. $72.7 \%$ were male and $27.3 \%$ were female, which was almost similar to our study. Similar result was also found by Mohandas et al.[13] However, few have reported 5:1 male: female ratio, when they detected Cryptosporidium infection in 60 renal transplant patients.[15,16]

In this study, On direct wet mount preparation only were $1 / 23$ Oocysts of Cryptosporidium could be detected as compared to that after concentration 2/23. On applying special staining method (Kinoyun's modified acid fast stain), 9 Cryptosporidium and this difference was significant. A similar finding have been reported by Wiwanitkit $\mathrm{V}$ et al and Ziring D et al were found $50 \%, 48 \%$ of the total subjects infected with intestinal protozoa respectively.[17,18] Nomir et al reported that after concentration there was increase from $27 \%$ to $56 \%$ by concentration method. Similarly on applying special staining methods for the identification of oppurtunistic parasitic pathogen diagnostic yield increased to 55.5\%.[19]

In the present study mixed infection was detected in 2/113 cases. One with Cryptosporidium and larva of Strongyloid and another one with Cryptosporidium and Isospora. This is important because even if we get one organism in sample it must be thoroughly screened, otherwise patient will not be completely cured.

Cryptosporidium antigen in stool was detected by ELISA in 23/113 (21\%) as compared to microscopy in 13/113 $(10.6 \%)$ and the difference was statistically significant $(\mathrm{p}<0.025)$. Sensitivity, specificity, PPV and NPV of antigen detection was $84.62 \%, 88 \%, 47.83 \%, 97.7 \%$ respectively. Two samples were microscopically positive but negative antigen detection. There could be several reasons for the discrepancy in results between the different techniques detecting oocysts in feces. In faecal samples, it is probably also an advantage to test for a soluble antigen that may be present more uniformly in the feces than to detect oocysts which are intermittently excreted. Monoclonal antibodies produced against $C$. parvum are generally considered to be genus specific rather than species specific and till date there has been no reports on monoclonal antibodies that can be used to differentiate between oocysts of different species.[19,20] In rest nine cases microscopy and antigen detection both were positive. Antigen detection picked by additional 12 cases which were microscopically negative. This may be due to the fact the sensitivity of antigen detection is very high $30 \mathrm{ng} / \mathrm{ml}$ as compared to microscopy. Our results are in agreement with the finding of Bialek Ralf et al who reported sensitivity of ELISA of Cryptosporidium was 94\% in his study and found antigen detection superior to microscopy.[21] But this is not in agreement with the finding of Nomir et al who did not find it to be much superior to microscopy.[18]

The present study confirmed the high prevalence of Cryptosporidium antigen in fecal samples of immunocompromised patients in the region. As no chemotherapeutic agents have yet proven, especially in immunosuppressed patients, therefore our results highlight the importance of preventive intervention in these groups.

ELISA showed high sensitivity and specificity with reliable results for Cryptosporidiumantigen detection in stool [23]. In addition cryptosporial antigens may be present in stool without existence the whole oocysts [24], therefore its application in routine diagnosis and even large-scale epidemiological surveys is recommended.

\section{CONCLUSION}

On the bases of the observation in the present study, Most of the patients infected with HIV and those who undergo renal transplantation are young. Male are at higher risk.Opportunistic parasitic pathogens are common in immunocompromised patients. Oppurtunistic parasitic pathogen are often missed on routine stool examination. Strong clinical suspicion and special staining methods must be used for their diagnosis. Cryptosporidium is most common opportunistic pathogen.Cryptosporidium commonly presents as chronic diarrhoea.Most of these parasites are detected when the CD4 count is low ( $200 \mathrm{cell} / \mu \mathrm{l})$. So immunocompromised patient with low CD4 count must be screened for oppurtunistic parasitic pathogen. Antigen detection in stool is a potentially useful method for the diagnosis of Cryptosporidium.

\section{References}

[1]. Gerald DS, Larry SR. Foundations of parasitology. 7th. Mc Graw Hill Companies; 2006. pp. 141-42.

[2]. Havelar A, Boonyakarnkul T, Cunliffe D, et al. Guidelines for drinking water qulity, water born pathogens. Geneva: WHO; 2003.

[3]. Abubakar I, Aliyu SH, Arumugam C, Usman NK, Hunter PR. Treatment of cryptosporidiosis in immunocompromised individuals: systemic review and meta analysis. Br J Clin Phamacol.2007;63(4):387-93. [PMC free article] [PubMed]

[4]. Mumtaz S, Ahmed J, Ali L, Frequency of Cryptosporidium infection in children under five years of age having diarrhoea in the North West of Pakistan, African Journal of Biotechnology, 2010; 9(8): 1230-35. 
[5]. Shrihari Narayan et. al. The Prevalence of Cryptosporidiosis in HIV Seropositive individuals and detection of Cryptosporidium parvum Oocysts by Modified Ziehl-Neelsen staining, Giemsa staining and Sheather sugar flotation technique in a Tertiary Care Hospital, JPBMS, 2011, 12 (09)

[6]. SitaraSwarna RA, PremiSankaran, Gagandeep Kang, Cryptosporidium species in HIV infected individuals in India: An overview, The National Medical Journal of India, 2008; 21(4):178-84.

[7]. Mumtaz S, Ahmed J, Ali L, Frequency of Cryptosporidium infection in children under five years of age having diarrhoea in the North West of Pakistan, African Journal of Biotechnology, 2010; 9(8): 1230-35.

[8]. Ortolani EL, Standardization of the modified Ziehl-Neelsen technique to stain oocysts of Cryptosporidium species, Rev Brazil Journal Vet Parasitol, 2000; 9(1): 29-31. 6.Karyakarte R, Damle A, Medical Parasitology, In: Other members of Phylum Apicomplexa, Chapter 6, Arunbhasen Book and Allied(P) Ltd, Kolkata; 2005:91. 9.MacPherson DW, McQueen R, Cryptosporidiosis: Multiattribute evaluation of six diagnostic methods, Journal of Clinical Microbiology, 1993; 31(2): 198-202.

[9]. Karyakarte R, Damle A, Medical Parasitology, In: Diagnostic methods in parasitology, Chapter 12, Arunbhasen Book and Allied (P) Ltd, Kolkata; 2005: 215.

[10]. Garcia LS, Diagnostic Medical Parasitology, In: Intestinal Protozoa (Coccidia and Microsporidia) and Algae, 4th Ed, ASM Press, USA; 2001:68. 